\title{
Functional intrinsic and extrinsic apoptotic pathways in human fetal mesenchymal stem cells
}

\author{
Cell Death and Differentiation (2005) 12, 1439-1441. doi:10.1038/sj.cdd.4401641; published online 17 June 2005
}

\author{
Dear Editor,
}

Nonembryonic stem cell populations have now been identified in most fetal and adult tissues. Not only can they act as a reservoir of pluripotent cells for repair or regeneration of the tissue where they reside, but they may also have wider potential. In the bone marrow, two stem cell populations (haematopoietic and mesenchymal) have been identified. Mesenchymal stem cells (MSC) are highly proliferative cells that can be maintained in culture and readily differentiated in vitro into mesodermal cell types (fat, bone, cartilage and muscle). ${ }^{1}$ While it has been shown that MSC can participate in the repair of mesodermal tissues, such as bone in osteogenesis imperfecta ${ }^{2}$ and heart muscle in cardiac ischaemia, ${ }^{3,4}$ this effect is limited as very few donor cells survive beyond a week. $^{3}$ The transgenic expression of antiapoptotic AKT in MSC used to repair a model of cardiac ischaemia resulted in a considerable improvement in myocardial volume, ${ }^{5}$ suggesting that a major obstacle to successful tissue repair is poor survival of stem cell grafts.

Recently, we have isolated a population of human MSC from first trimester fetal blood that are phenotypically similar to adult-derived bone marrow stromal cells and can be readily differentiated into fat, bone and cartilage. ${ }^{6}$ Preliminary data also suggest that fetal MSC can be differentiated along myogenic and neural lineages, and so represent a ready source of human multipotent cells. Although it might be expected that highly proliferative stem cells would be equipped with efficient death machinery, there is little published work on apoptotic signalling pathways in MSC or bone marrow-derived stromal cells, ${ }^{7-9}$ and even fewer studies of apoptosis in other stem cells. ${ }^{10}$

Since apoptosis is a major cause of graft failure, we hypothesised that there would be robust and functional intrinsic and extrinsic apoptotic signalling pathways in naïve human MSC. Undifferentiated fetal MSC were exposed to two apoptotic stimuli, staurosporine (SSP) or CD95/Fas, and the activation of the intrinsic (mitochondrial) and extrinsic (death receptor) pathways was then investigated. We report the presence and activity of both death pathways and demonstrate the sensitivity of MSC to these two apoptotic stimuli.

We first assessed MSC viability following a $24 \mathrm{~h}$ exposure to SSP and found that cell death was triggered in a dosedependent manner with maximum toxicity observed at approximately $200 \mathrm{nM}$ SSP (Figure 1a). Dead cells displayed a typical apoptotic morphology (Figure 1b) and apoptosis was confirmed by TUNEL (Figure 1c, inset). In time-course experiments, MTT metabolism declined within $1 \mathrm{~h}$ of SSP addition and by $3.5 \mathrm{~h}$ had fallen by $40 \%$ compared to healthy controls (Figure 1c). The appearance of morphological changes consistent with apoptosis within minutes of exposure to SSP suggested an early commitment to apoptotic death in MSC. In order to examine this in more detail, fetal MSC were exposed transiently to SSP for defined times, washed thoroughly and then returned to complete medium for $24 \mathrm{~h}$ to allow surviving cells to recover. With only a 15 min exposure to SSP, $85 \%$ of fetal MSC were committed to apoptotic cell death (not shown).

To further study the mechanism of SSP-induced apoptosis, cytochrome $c$ localisation was determined in cells exposed to SSP. In contrast to punctate, mitochondrial staining of cytochrome $c$ in healthy cells, within $2 \mathrm{~h}$ of SSP treatment, diffuse cytoplasmic staining was apparent, indicating release of cytochrome $c$ into the cytosol (Figure 1d). At early time points, cytochrome $c$ release could be dissociated from subsequent nuclear pyknosis and fragmentation, although by $8 \mathrm{~h} 60 \%$ of cells were positive for cytosolic cytochrome $c$ (Figure 1d, graph) and many of these also contained pyknotic nuclei.

In time-course experiments, SSP-dependent processing of caspases 9 and 3 was confirmed by immunocytochemistry and Western blotting using primary antibodies specific for the cleaved fragments; significantly, caspase- 9 cleavage began before caspase 3 (Figure 1e) and preceded nuclear pyknosis and DNA fragmentation. Consistent with the absence of caspase-8 cleavage following exposure to SSP (data not shown), SSP-induced cell death could be attenuated by incubation with both caspase- 9 and pancaspase inhibitors in a dose-dependent manner (Figure 1f) while a selective caspase-8 inhibitor (IETD-fmk) had no protective effect (not shown). These results show that fetal MSC are very sensitive to apoptotic cell death via the mitochondrial pathway and confirm the importance of mitochondria in stem cell apoptosis, ${ }^{11}$ also demonstrated following leptin treatment of adult bone marrow stromal cells ${ }^{9}$ and the exposure of neural stem cells to oxidative stress. ${ }^{10,12}$

Having established that the intrinsic (mitochondrial) pathway for apoptotic death was functional in fetal MSC, we extended the study to explore the role of the extrinsic (death receptor) pathway. CD95/Fas ligation was chosen as a stimulus as this receptor has been detected on murine bone marrow stromal cells. ${ }^{8}$ The expression of CD95/Fas on human MSC was confirmed by Western blot analysis (not shown) and its surface expression was demonstrated to be highly polarised using immunocytochemistry (Figure 2a). We 
a
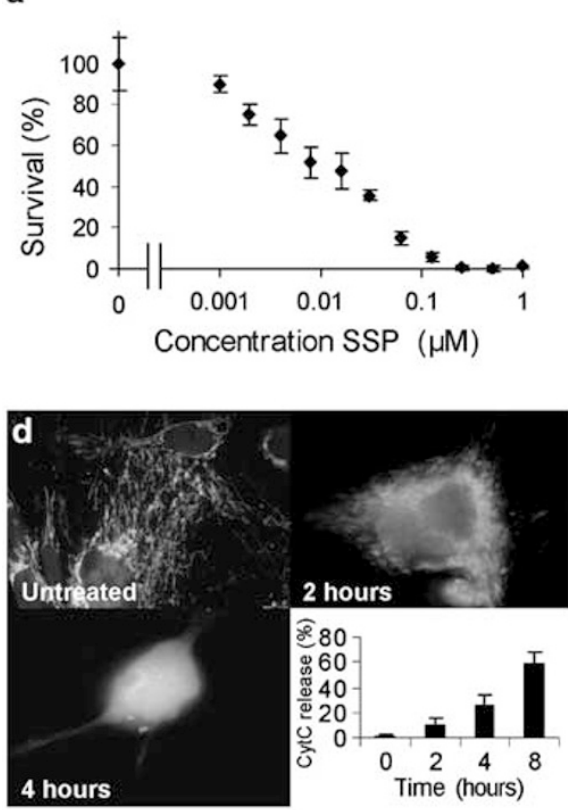

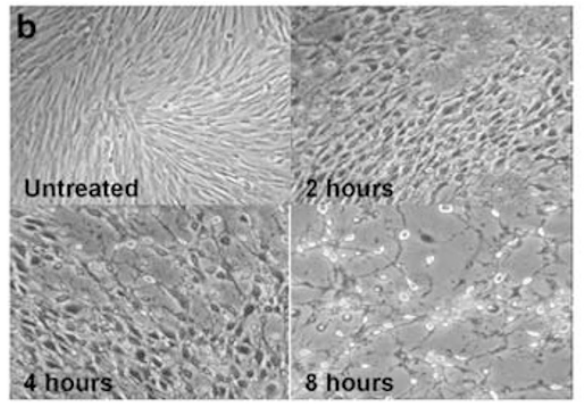

e

Caspase 9

Caspase 3

$\alpha$ tubulin

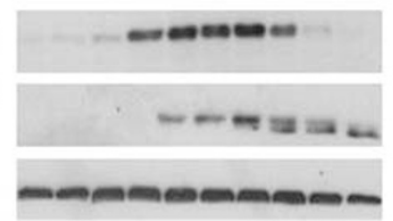

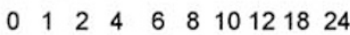

SSP exposure time (hours)

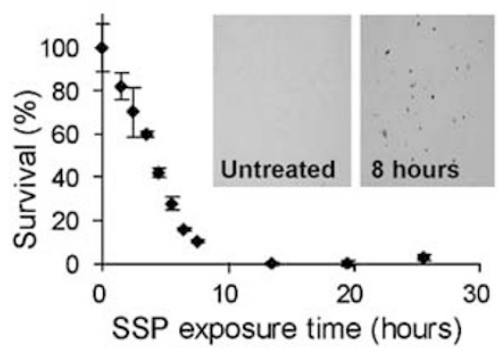

f

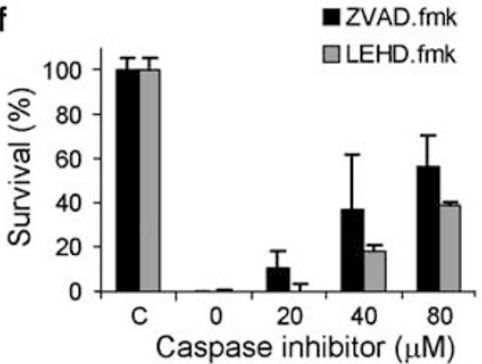

Figure 1 SSP triggers apoptosis in fetal MSC via the mitochondrial pathway. Fetal MSC were treated with increasing concentrations of SSP (in DMEM/0.5\% FCS) for $24 \mathrm{~h}$ and cell death assessed by MTT assay (a). In time-course experiments using $200 \mathrm{nM} \mathrm{SSP}$, cells adopted typical apoptotic morphology (b) and were all dead by $13 \mathrm{~h}$ (c). Apoptosis was confirmed by TUNEL (inset in c). (d) Summary of immunofluorescence studies of cytochrome clocalisation following exposure of human fetal MSC to SSP $(200 \mathrm{nM})$. In healthy cells, cytochrome $c$ (antibody supplied by BD Biosciences, Oxford, UK), detected with an FITC-labelled secondary antibody, is located in the mitochondria and stains in a punctate or reticular pattern. When cytochrome $c$ is released into the cytosol, staining becomes more diffuse. Note that cytochrome $c$ release occurs before nuclear pyknosis. In time-course experiments, cytochrome $c($ CytC) release was quantitated by fluorescence microscopy with data plotted as the mean $( \pm$ S.D.) of four data points (inset in (d)). The cleavage of caspases was determined by Western blotting with cleavage-specific antibodies. In time-course experiments, cleavage of caspase 9 was evident before caspase 3 by immunocytochemistry (f) using cleavage-specific antibodies (Cell Signalling, Hitchin, UK). Pretreatment of cells for $1 \mathrm{~h}$ prior to SSP treatment $(200 \mathrm{nM})$ with the cell-permeable pancaspase inhibitor ZVAD.fmk or the caspase-9 inhibitor Z-LEHD.fmk (Gentaur, Brussels, Belgium) protected against cell death determined by MTT assay at $24 \mathrm{~h}$. Data are the mean $( \pm$ S.D.) of four independent experiments

found that the adaptor molecule, FADD, was also present in fetal MSC, and that its expression was both cytosolic and nuclear (Figure 2b). The nuclear localisation of FADD is consistent with a recent report $^{13}$ and might explain the interaction of FADD with several nuclear proteins involved in apoptosis, including methyl-Cpg binding protein-4. ${ }^{14}$ Caspase 8 was also found to be expressed in human fetal MSC (not shown). However, the mere presence of these CD95/Fas signalling components does not prove the existence of an active extrinsic death pathway. Moreover, many Fas-expressing cells do not undergo death when Fas receptor is bound by its ligand, ${ }^{15}$ and alternative biological processes such as proliferation in T cells ${ }^{16}$ and fibroblasts, ${ }^{17}$ activation of NF$\kappa \mathrm{B}^{18}$ and neurite formation in primary sensory neurones ${ }^{19}$ have been attributed to Fas ligation.

In the present study, the addition of Fas ligand (FasL) to healthy fetal MSC did induce cell death by apoptosis, defined by cell morphology and TUNEL (not shown). In time-course experiments, an exposure time of $72 \mathrm{~h}$ was sufficient to kill more than half the MSC population. Moreover, there was a dose-dependent increase in apoptosis following exposure to FasL (Figure 2c) and these results were confirmed in viable cell counting experiments. FasL-induced death could be inhibited by the pancaspase inhibitor ZVAD.fmk and by the selective caspase-8 inhibitor IETD.fmk. Preincubation with either inhibitor afforded protection against FasL-induced apoptosis (Figure 2c). In contrast, caspase-9 inhibition with Z-LEHD.fmk at an effective dose (defined as $20-80 \mu \mathrm{M}$ by the SSP experiments) was not protective against FasL-induced death (data not shown). These findings indicate that extrinsic death receptor signalling is intact in human fetal MSC. These data contrast the observations in neural stem cells, where CD95/Fas is expressed although agonistic Fas antibody failed to induce apoptosis; the authors speculate that the extrinsic apoptosis pathway might be developmentally regulated. ${ }^{12}$

Understanding the mechanisms by which MSC undergo cell death may help in developing strategies to promote graft survival in stem cell therapy. In the present study, we have shown that primary human fetal MSC can undergo cell death by apoptosis triggered by two different stimuli - exposure to SSP or CD95/Fas ligation. The choice of these stimuli was deliberate: SSP was used as a stress stimulus known to activate the mitochondrial pathway in many other cell types and Fas ligation was used to test death receptor signalling (and may partly model immune-mediated cell death). This is the first study demonstrating functional mitochondrial and death receptor apoptosis pathways in primary naïve human fetal MSC. A detailed understanding of apoptotic signalling in these cells will be helpful in designing strategies for increasing the survival of stem cell grafts. 

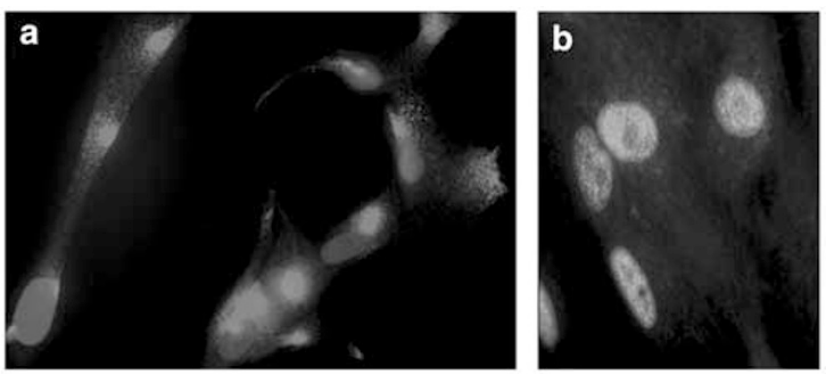

C

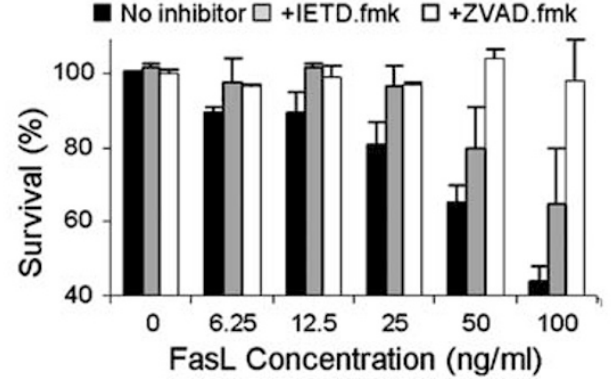

Figure $2 \mathrm{CD} 95 /$ Fas ligand (FasL) triggers apoptosis in fetal MSC via the extrinsic, death receptor pathway. CD95/Fas was detected by immunocytochemistry with the pattern appearing polarised on untreated cells (a). FADD was present in the cytosol, but staining also colocalised with the nucleus (b). The Fas pathway was functional and apoptosis was induced over $72 \mathrm{~h}$ in a dosedependent manner (c) using crosslinked recombinant FasL (Alexis Corporation UK, Nottingham, UK). Over this time course, CD95/Fas-dependent apoptosis could be inhibited using the pancaspase inhibitor ZVAD.fmk $(40 \mu \mathrm{M})$ and attenuated by caspase-8 inhibitor (IETD.fmk, $10 \mu \mathrm{M}$ ) (c). Data are the mean $( \pm$ S.D.) of four data points in a representative experiment

\section{NL Kennea ${ }^{1}$, C Stratou $^{1}$, A Naparus $^{1}$, NM Fisk $^{1}$ and H Mehmet $^{*, 1}$}

1 Institute of Reproductive and Developmental Biology, Faculty of Medicine, Imperial College London, Hammersmith Hospital Campus, Du Cane Road, London W12 ONN, UK

* Corresponding author: $\mathrm{H}$ Mehmet, Institute of Reproductive and Developmental Biology, Division of Paediatrics, Obstetrics and Gynaecology, Faculty of Medicine, Imperial College London, Hammersmith Hospital, Du Cane Road, London W12 ONN, UK. Tel: + 44207594 2190; Fax: + 44207594 2192; E-mail: h.mehmet@imperial.ac.uk

1. Pittenger et al. (1999) Science 284: 143-147

2. Horwitz et al. (1999) Nat. Med. 5: 309-313

3. Toma et al. (2002) Circulation 105: 93-98

4. Orlic et al. (2001) Nature 410: 701-705

5. Mangi et al. (2003) Nat. Med. 9: 1195-1201

6. Campagnoli et al. (2001) Blood 98: 2396-2402

7. Li et al. (2004) Br. J. Haematol. 127: 326-334

8. Lepri et al. (1998) Exp. Hematol. 26: 1202-1208

9. Kim et al. (2003) J. Biol. Chem. 278: 21920-21929

10. Sleeper et al. (2002) Cell Death Differ. 9: 1377-1378

11. Green and Reed (1998) Science 281: 1309-1312

12. Tamm et al. (2004) Eur. J. Neurosci. 19: 2613-2621

13. Gomez-Angelats and Cidlowski (2003) Cell Death Differ. 10: 791-797

14. Screaton et al. (2003) Proc. Natl. Acad. Sci. USA 100: 5211-5216

15. Ahn et al. (2001) J. Biol. Chem. 276: 47100-47106

16. Alderson et al. (1993) J. Exp. Med. 178: 2231-2235

17. Aggarwal et al. (1995) FEBS Lett. 364: 5-8

18. Rensing-Ehl et al. (1995) J. Inflamm. 45: 161-174

19. Desbarats et al. (2003) Nat. Cell Biol. 5: 118-125 